\title{
ARTICLE
}

\section{Development of improved cubic spline method for synthesizing the axial power distribution in a nuclear reactor}

\author{
Byung Hee Won, Kyung-O Kim and Jong Kyung Kim* \\ Department of Nuclear Engineering, Hanyang University, Seoul, Korea
}

\begin{abstract}
The improved cubic spline method using new function sets and basis functions is developed for accurately predicting the Axial Power Distribution (APD) in a nuclear reactor. The improved function sets and basis functions are derived by analyzing 3,000 APDs from measured and calculated data for the OPR-1000 reactor (PWR). When comparing conventional and improved methods, APDs produced by improved method represent an average Root Mean Square (RMS) error of $1.68 \%$, on the basis of reference data, whereas the other has an average RMS error more than $2.40 \%$. Especially, the performance improvement is remarkably shown in APDs classified as the center peak and saddle types. The average RMS errors in the center peak and saddle types decrease by 21.03 and $51.36 \%$ respectively. The cubic spline method established in this study would complement the conventional method and enhance the accuracy to within the average RMS error of $1.80 \%$ in center peak, flat, and saddle types. It is expected that the unnecessary reactor trip by inaccurate prediction of power distributions would be reduced as well as improve the safety and economics of nuclear power plant by performance improvement of the cubic spline method in CPCS.
\end{abstract}

Keywords: Core Protection Calculator System; ex-core detector; cubic spline method; Axial Power Distribution

\section{Introduction}

The Core Protection Calculator System (CPCS) should be employed to protect nuclear power plants from the occurrence of the Departure from Nucleate Boling Ratio (DNBR) and Local Power Density (LPD). To obtain the information of the DNBR and LPD, the axial power distribution is predicted by signals obtained from ex-core detectors. Also, the accuracy of this axial power distribution is mainly influenced by the function sets and basis functions in the cubic spline method [1]. The existing cubic spline method has employed only one function set and one basis function regardless of the type of the axial power distributions. However, the use of these sets would not cover various power shapes, such as the center peak, flat, and the saddle types, occurred over the reactor operation period. Thus, many countries operating nuclear power plants have focused on an accurate prediction of the axial power distribution by the cubic spline method through the enlargement of those functions.

In this study, the cubic spline method used in the CPCS having the three ex-core detectors (top, middle, and bottom) was investigated to apply the conventional system immediately. Many function sets $(18,424$ cases) and basis functions (49 cases) were introduced during

*Corresponding author. Email: jkkim1@hanyang.ac.kr the synthesis of axial power distributions for the OPR-1000 reactors. From these analyses, the improved function sets and basis functions were established to accurately predict various power shapes such as center peak, flat, and saddle types. Their performances were evaluated through the difference (RMS error) between the reference data and the consequences using the conventional and improved cubic spline method.

\section{Cubic spline method}

The cubic spline method synthesizes the axial power distribution by summing the cubic spline basis functions multiplied by the amplitude coefficients as given in Eq. (1).

$$
P(z)=\sum_{i=1}^{7} a_{i} \mu_{i}(z)
$$

$$
\text { where } \begin{aligned}
P(\mathrm{z}) & =\text { Axial power disribution at location } \mathrm{z} \\
a_{i} & =\text { Amplitude coefficients } \\
\mu_{i}(\mathrm{z}) & =\text { Cubic spline basis functions }
\end{aligned}
$$

It is noted that seven cubic spline basis functions composed of the $3^{\text {rd }}$ order polynomial are arranged on the axial core height as shown in Figure 1, and these are 
vertically expanded by multiplying the amplitude coefficients. The axial power distribution can finally be predicted by summing the expanded basis functions.

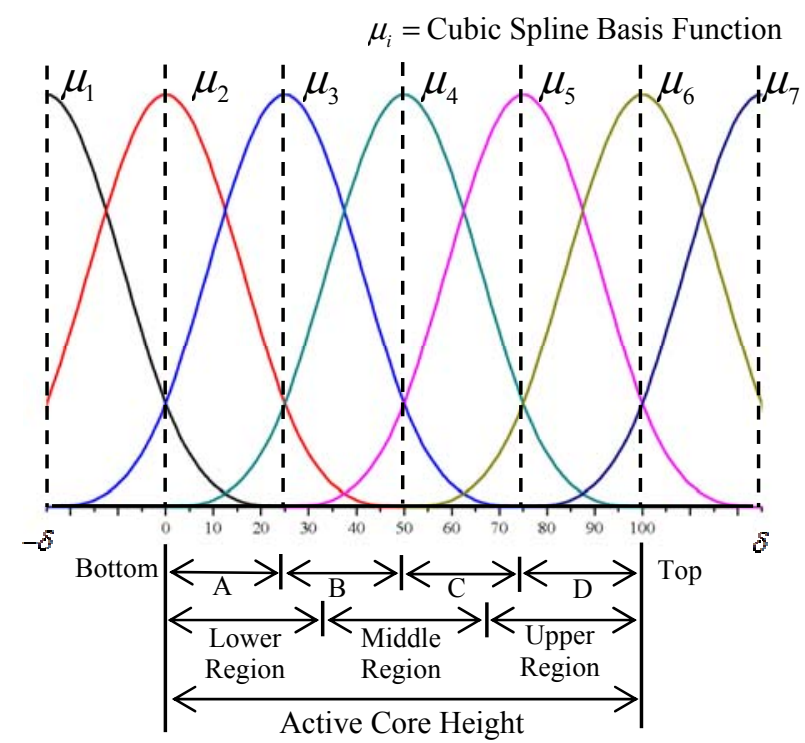

Figure 1. Schematic of Cubic Spline Method

To synthesize the power shape, it is necessary to determine the amplitude coefficients by the signals of ex-core detectors located outside of the reactor vertically. Since the objective of this study was to improve the cubic spline method itself, the values calculated from the reference data (i.e., axial power distributions) were employed instead of original signals obtained by ex-core detectors. To evaluate the results obtained by this study, Root Mean Square (RMS) error, the difference between synthesized power distribution and reference data, was introduced to compare the conventional method. The equation of RMS error is given in Eq. (2).

RMS error $(\%)=\sqrt{\frac{\sum_{i=1}^{20}\left(E_{i}\right)^{2}}{20}}$

where

$E_{i}=\left(\frac{P\left(z_{i}\right)_{\text {Synthesis }}-P\left(z_{i}\right)_{\text {Reference }}}{P\left(z_{i}\right)_{\text {Reference }}}\right) \times 100$
$P\left(z_{i}\right)_{\text {Synthesis }} \quad \begin{aligned} & : \text { Power made by synthesis method } \\ & \text { at location } z_{i}\end{aligned}$
$P\left(z_{i}\right)_{\text {Reference }}: \begin{aligned} & : \\ & \text { Power in reference data } \\ & \text { at location } z_{i}\end{aligned}$

\section{Performance evaluation of improved function sets}

The cubic spline function set is one of the factors which affects the performance for synthesizing the axial power distribution. This set determines where each interval (A, B, C, and D) is assigned to axial core height. That is, the cubic spline function set indicates the position which each of seven basis functions is arranged. The different arrangements of function sets affect synthesis performance. In the conventional method, the active core height is divided into twenty nodes and these nodes are distributed among the four intervals. Furthermore, the function sets have a condition that the sum of A and B interval should be equal to the sum of C and D symmetrically. 2-8-8-2 function set, which divides active core height into four intervals in the ratio of 10:40:40:10 as shown in Figure 2, is currently used in the existing method to synthesize all axial power distributions regardless of its shapes. This sometimes causes poor performance because the axial power distributions are presented as many forms, such as center peak, flat, and saddle, according to the burnup.

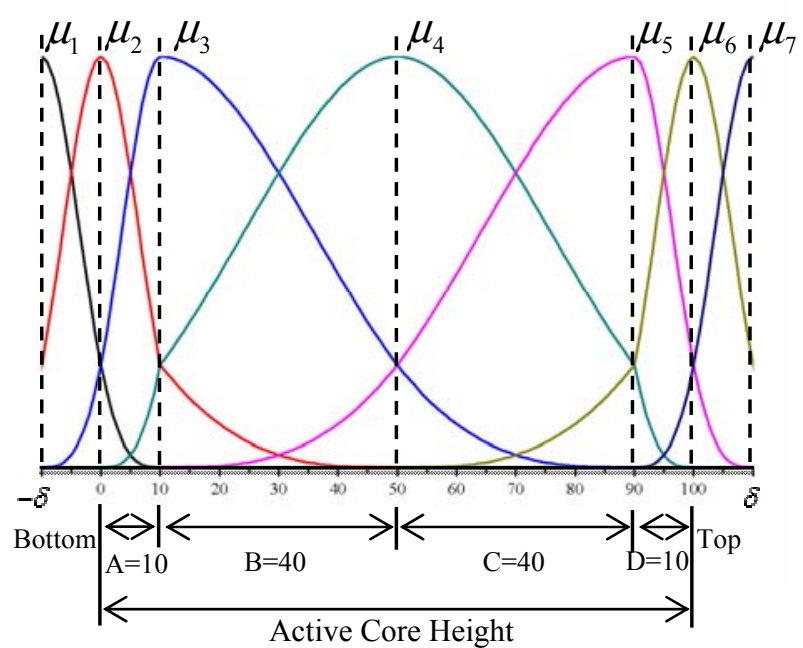

Figure 2. Arrangement of Basis Functions by 2-8-8-2 Cubic Spline Function Set

In this study, the active core height was divided into fifty nodes and these nodes were distributed to four intervals with symmetric and asymmetric function sets. This method is suitable for evaluating the more function sets without the symmetric condition in comparison with the conventional one. To conduct efficient calculation, this study was performed using Matlab ${ }^{\mathrm{TM}}$ program. First, 18,424 types of function sets were applied to each reference data and one was selected as an optimal function set. The result indicated that the optimal function sets gave better performance than the conventional one (2-8-8-2 function set), as shown in Figure 3. In particular, the asymmetric function sets which had not been considered as conventional were evaluated as the best performing set in many reference data. In Table 1, the asymmetric function sets were presented as optimum in more than ninety percent of the reference data. Additionally, the improved function set which gave the best performance according to each type of the axial power shapes was evaluated. Table 2 shows the results that synthesis performance was ameliorated by the improved function sets, compared to the conventional function set. It was indicated that 4-23-19-4 and 4-12-31-3 function sets performed well at 
center peak and saddle types. But the improved function set, 5-17-23-5 function set, applied at flat type was not significantly different. In conclusion, substituting 2-8-8-2 function set for the improved function sets in center peak and saddle types may decrease RMS error significantly.

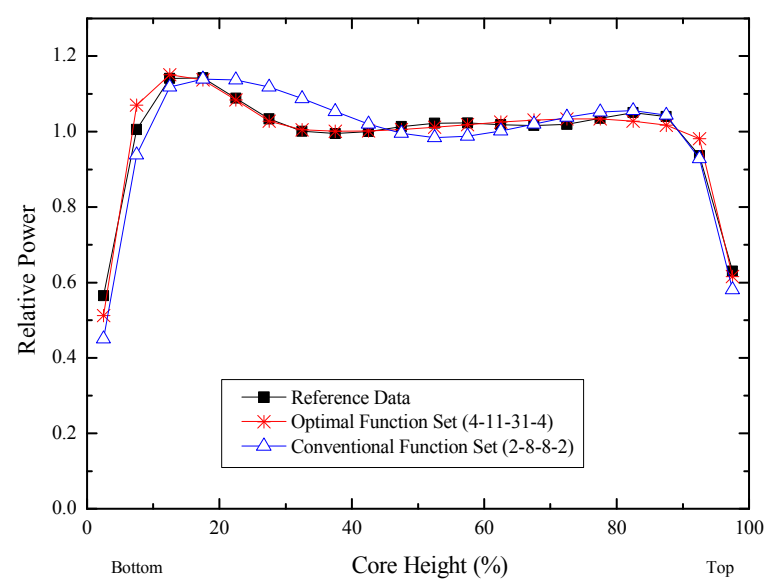

Figure 3. Synthesis Result of Axial Power Distribution

Table 1. Selection Frequency of Asymmetric Function Sets

\begin{tabular}{cccc}
\hline Type & $\begin{array}{c}\text { Number of } \\
\text { Reference Data }\end{array}$ & $\begin{array}{c}\text { Selection of } \\
\text { Asymmetric } \\
\text { Function Sets }\end{array}$ & Ratio \\
\hline Center & 1000 & 922 & $92.2 \%$ \\
\hline Flat & 1000 & 911 & $91.1 \%$ \\
\hline Saddle & 1000 & 955 & $95.5 \%$ \\
\hline
\end{tabular}

Table 2. Comparison of Synthesis Results by Conventional and Improved Function Sets

\begin{tabular}{cccc}
\hline Type & $\begin{array}{c}\text { Conventional } \\
\text { Function Set }\end{array}$ & $\begin{array}{c}\text { Improved } \\
\text { Function Set }\end{array}$ & $\begin{array}{c}\text { Decreasing Rate } \\
\text { of RMS error }\end{array}$ \\
\hline Center & $2-8-8-2$ & $4-23-19-4$ & $\begin{array}{c}21.30 \% \\
(2.14 \rightarrow 1.69)\end{array}$ \\
\hline Flat & $2-8-8-2$ & $5-17-23-5$ & $\begin{array}{c}0.61 \% \\
(1.56 \rightarrow 1.55)\end{array}$ \\
\hline Saddle & $2-8-8-2$ & $4-12-31-3$ & $\begin{array}{c}34.67 \% \\
(3.68 \rightarrow 2.41)\end{array}$ \\
\hline
\end{tabular}

* The use of the convention basis function

\section{Performance evaluation of improved basis functions}

The cubic spline basis function is another factor which affects the performance for synthesizing the axial power distribution. Eq. (3) shows detailed structure of the basis function. It consists of two functions $\left(f_{1}(\eta), f_{2}(\eta)\right)$ with the condition that it must be a continuous function at each break point $\left(z_{i-2}, z_{i-1}, z_{i}, z_{i+1}, z_{i+2}\right)$. The shape of the basis function is determined by a constant $\left(A_{1}\right)$. Figure 4 represents that the various basis functions have different shape, wide or narrow, according to $A_{1}$. If the value of $A_{1}$ is smaller than the value $\left(A_{1}=0.25\right)$ in the conventional basis function, the shape of basis functions has narrow width. On the other side of the value of $A_{1}$, the shape has wide width.

$$
\begin{gathered}
\mu_{i}(z)=\left\{\begin{array}{lll}
f_{1}\left(\eta_{1}\right) & \text { for } & z_{i-2} \leq z \leq z_{i-1} \\
f_{2}\left(\eta_{2}\right) & \text { for } & z_{i-1} \leq z \leq z_{i} \\
f_{2}\left(\eta_{3}\right) & \text { for } & z_{i} \leq z \leq z_{i+1} \\
f_{1}\left(\eta_{4}\right) & \text { for } & z_{i+1} \leq z \leq z_{i+2} \\
0 & \text { for } & z<z_{i-2} \text { or } z>z_{i+2}
\end{array}\right. \\
\text { where } \begin{array}{ll}
\eta_{1}=\frac{z-z_{i-2}}{z_{i-1}-z_{i-2}}, & \eta_{2}=\frac{z-z_{i-1}}{z_{i}-z_{i-1}} \\
& \eta_{3}=\frac{z_{i+1}-z}{z_{i+1}-z_{i}}, \quad \eta_{4}=\frac{z_{i+2}-z}{z_{i+2}-z_{i+1}} \\
& f_{1}(\eta)=A_{1} \eta^{3} \\
& f_{2}(\eta)=\left(-2+5 A_{1}\right) \eta^{3}+\left(3-9 A_{1}\right) \eta^{2}+3 A_{1} \eta+A_{1}
\end{array}
\end{gathered}
$$

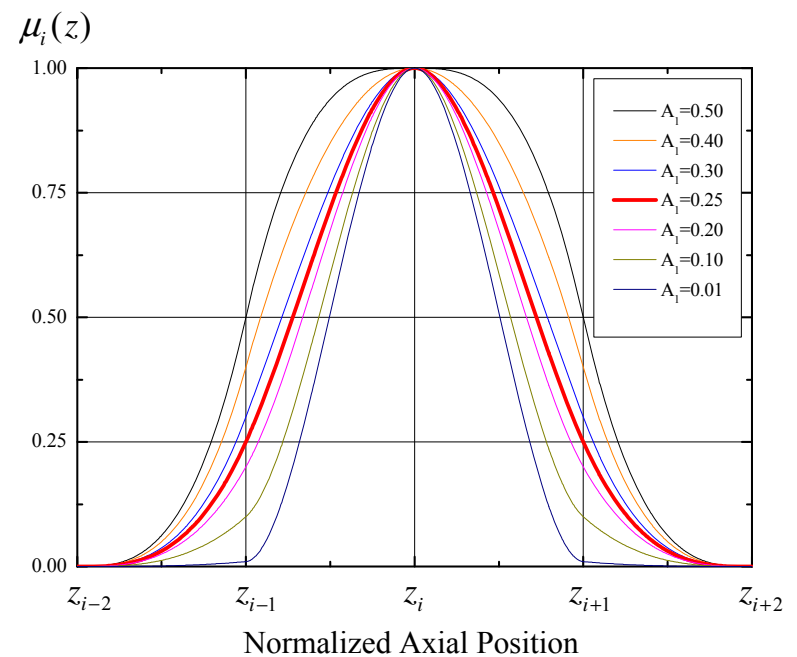

Figure 4. Cubic Spline Basis Function

In the conventional method, only one type of base function $\left(A_{1}=0.25\right)$ was used to predict all axial power shapes regardless of its types. This occasionally leads to inaccurate prediction, for there are various forms of power distribution. In this study, forty-nine types of basis functions $\left(A_{1}=0.01 \sim 0.50\right)$ having narrow or wide shapes were applied to the synthesis process according to the types of axial power distributions with 18,424 function sets. Nine types of basis functions brought better results in center peak type and four types of basis functions showed better performance in flat type. However, new basis functions in center peak and flat type decreased RMS error by $1.09 \%$ at the most when compared with conventional basis function. It means that the performance improvements resulted from the new basis functions were minor in center peak and flat type. 
Table 3. Comparison of Synthesis Results by Conventional and Improved Method

\begin{tabular}{|c|c|c|c|c|c|}
\hline Type & $\begin{array}{l}\text { Conventional } \\
\text { Function Set }\end{array}$ & $\begin{array}{c}\text { Conventional } \\
\text { Basis Function }\end{array}$ & $\begin{array}{l}\text { Proposed } \\
\text { Function Set }\end{array}$ & $\begin{array}{c}\text { Proposed } \\
\text { Basis Function }\end{array}$ & $\begin{array}{l}\text { Decreasing Rate } \\
\text { of RMS error }\end{array}$ \\
\hline Center & & & $4-23-19-4$ & - & $\begin{array}{c}21.30 \% \\
(2.14 \rightarrow 1.69)\end{array}$ \\
\hline Flat & $2-8-8-2$ & $A_{1}=0.25$ & - & - & - \\
\hline Saddle & & & $3-16-28-3$ & $A_{1}=0.01$ & $\begin{array}{c}51.36 \% \\
(3.68 \rightarrow 1.79)\end{array}$ \\
\hline
\end{tabular}

While, in saddle type, the performance improvements were presented in all basis functions having a narrower shape than the conventional one. Figure 5 shows RMS errors according to the type of basis functions in saddle types. The function sets were selected as the optimal ones among 18,424 function sets in each basis function. It was found that, as the shape of basis function has narrower shape, synthesis performance gets better result. For the basis functions, narrow width is suitable for predicting the axial power shapes classified as the saddle type. From the analysis in Figure 5, 3-16-28-3 function set and the basis function $\left(A_{1}=0.01\right)$ were selected as the optimal combination for predicting the axial power distributions having the saddle type.

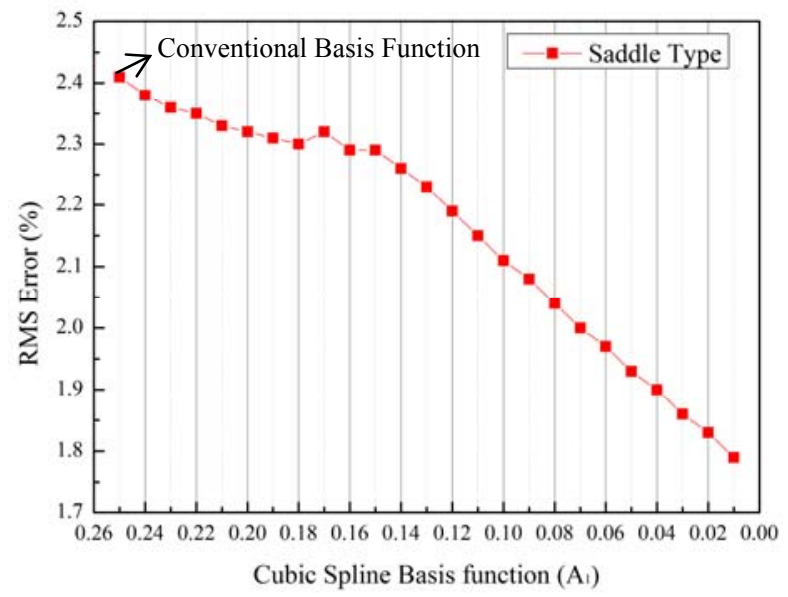

Figure 5. RMS Errors by Basis Functions in Saddle Type

\section{Conclusions}

This study was conducted to improve the accuracy of predicting axial power distribution. 18,424 function sets and forty-nine types of basis functions were evaluated to find the optimal ones. In the analysis of the function sets, the symmetric function sets such as $2-8-8-2$ is not suitable whereas many asymmetric ones are appreciated as the optimal function sets. The improved function sets were also suggested for each type (center peak, flat, saddle type). 4-23-19-4 and 3-16-28-3 function sets presented as asymmetric ones show good performance in center peak and saddle types respectively. The cubic spline basis function $\left(A_{1}=0.01\right)$ having narrow shape in comparison to the conventional one improves synthesis performance of axial power distributions in saddle type.
In conclusion, the cubic spline method for accurate prediction of the axial power distributions was finally proposed as shown in Table 3.

It was concluded that the cubic spline method using new function sets and basis functions, suggested in this study, would accurately predict the power distributions in reactor core. The CPCS could obtain more accurate axial power distributions to monitor the DNBR and LPD. From these performances, the safety of nuclear power plant is assured and the economics is also improved by preventing unwanted reactor trip.

\section{Acknowledgements}

This work was supported in part by Basic Science Research Program through the NRF of Korea funded by Ministry of Education, Science and Technology of Korea [MEST] (2012-001545), NHDD Project coordinated by Korea Atomic Energy Research Institute funded by MEST (2012-025679), and Innovative Technology Center for Radiation Safety (iTRS) at Hanyang University.

\section{References}

[1] W. K. In and B. O. Cho, On-line Core Axial Power Distribution Synthesis Method from In-core and Ex-core Neutron Detectors, KAERI/TR-1415, Korea Atomic Energy Research Institute, (1999).

[2] W. K. In, H. K. Yoo, G. S. Auh, C. C. Lee and S. H. Kim, Application of cubic spline synthesis in on-line core axial power distribution monitoring, Journal of Korean Nuclear Society. 23 (1991), pp. 316-320.

[3] B. S. Koo, C. C. Lee and S. Q. Zee, Improvement of the cubic spline function sets for a synthesis of the axial power distribution of a core protection system, Transaction of KNS Autumn Meeting, Gyeongju, Nov. 2-3, (2006).

[4] B. S. Koo, J. Y. Cho and J. S. Song, Evaluation of Applicability for the Core Protection Method with 4-Channel CEA Positions to OPR-1000 Plants, KAERI/TR-3580, Korea Atomic Energy Research Institute, (2008). 